(C) 2015 IEEE. Personal use of this material is permitted. Permission from IEEE must be obtained for all other uses, in any current or future media, including reprinting/republishing this material for advertising or promotional purposes, creating new collective works, for resale or redistribution to servers or lists, or reuse of any copyrighted component of this work in other works. 


\title{
Simultaneous control of dielectric charge and device capacitance in electrostatic MEMS
}

\author{
S. Gorreta, Student Member, IEEE, J. Pons-Nin and M. Dominguez-Pumar, Senior Member, IEEE \\ Micro and Nano Technologies Group, Electronic Engineering Department, Universitat Politècnica de Catalunya, Spain
}

\begin{abstract}
This work presents a double closed-loop for controlling simultaneously the net dielectric charge and the device capacitance in contactless electrostatic MEMS devices. The first loop controls the net charge trapped in the dielectric layer by continuously monitoring the horizontal displacement of the $\mathrm{C}-\mathrm{V}$ characteristic and applying bipolar actuation voltages to keep such net charge at the target value. The second loop adapts the actuation voltages so that the measured capacitance matches a desired value, while maintaining the primary control of charge.
\end{abstract}

Index Terms-Dielectric charging, MEMS, charge control, positioners, varactors.

\section{INTRODUCTION}

Dielectric charging is an important reliability issue for the use of electrostatic MEMS devices in many applications [1]. This phenomenon, caused by the actuation of the device, produces effects such as shifting of the $\mathrm{C}-\mathrm{V}$ curve and even device failure due to stiction. It is widely assumed that the sign of the charge injected into the dielectric depends on the polarity of the voltage applied. For instance in a device working in the below pull-in (or contactless) state, net negative charge is injected when applying positive voltages, whereas net positive charge appears when applying negative voltages. These changes of the net dielectric charge can be detected as horizontal shifts of the C-V.

To mitigate the effect of dielectric charging, some open-loop strategies have been proposed [2]. These strategies alternate the polarity of the actuation voltage following a periodic pattern in order to compensate the total injected charge. Although these techniques have demonstrated to increase the lifetime of devices, they cannot adapt to changes in the charge dynamics and therefore they may be not effective at long term. Regarding this, closed-loop charge control strategies based on sigma-delta modulation have been proposed [3], [4]. These controls periodically monitor the horizontal shift of the $\mathrm{C}-\mathrm{V}$, thus the net dielectric charge, by performing quasi-differential capacitance measurements and actuating the device with bipolar voltage waveforms, allowing to set the net dielectric charge to a target value.

Additionally, non-uniform distributions of charge and environmental variations can also cause vertical displacement of the $\mathrm{C}-\mathrm{V}$ [5]. For instance, it has been reported that a nonuniform charge distribution on the dielectric surface produces additional electrostatic force between the MEMS electrodes, even when the total charge is zero. This phenomenon, modelled in [6], together with changes in temperature [7] or humidity [8] can explain why the device capacitance may vary even when charge control is being applied. This may imply that although the net dielectric charge is being controlled, a constant device capacitance is not guaranteed.

This work introduces a double-loop control strategy which allows to maintain both a target level of net dielectric charge and a target capacitance value. The first loop performs a sigmadelta control of charge: it indirectly monitors the total charge and actuates the device with waveforms composed of two voltages, $V^{+}>0$ and $V^{-}<0$. The second loop implements a method that dynamically varies the value of $V^{+}$and $V^{-}$to keep constant the device capacitance. This new control method is described in section II and verified through experiments in section III.

\section{DESCRIPTION OF THE CONTROL METHOD}

As discussed above, the method proposed here allows to maintain both a desired amount of net dielectric charge and a certain target capacitance. The net dielectric charge can be inferred from the voltage shift of the $\mathrm{C}-\mathrm{V}$ curve, $V_{s h}$. To obtain this value, a quasi-differential capacitance measurement is made at each sampling time $T_{S}$ by applying to the device either one of the actuation waveforms, or symbols, BIT0 ( $V^{-}$ applied during most of the sampling period) or BIT1 (the same for $V^{+}$) shown in Fig. 1. Capacitance measurements performed at symbol times $(1-\delta) T_{S}$ and $T_{S}$ allow to obtain the quasi-differential capacitance $\Delta C=C^{+}-C^{-}$, where $C^{+}$ and $C^{-}$are the device capacitances measured when applying $V^{+}$and $V^{-}$, respectively. Taking into account that for voltages below pull-in the $\mathrm{C}-\mathrm{V}$ can be approximated by a parabolic function [3], [9], $V_{s h}$ can be obtained at $t=n T_{S}$ as:

$$
V_{s h}(n)=\frac{\alpha\left(\left(V^{+}\right)^{2}-\left(V^{-}\right)^{2}\right)-\Delta C(n)}{2 \alpha\left(V^{+}-V^{-}\right)}
$$

where the coefficient $\alpha$ is assumed constant. This coefficient can be obtained from a $\mathrm{C}-\mathrm{V}$ fitting.

The block diagram of Fig. 2 describes the double-loop control proposed. It allows to set the device capacitance to a target value $C_{t h}$, against potential vertical displacement of the $\mathrm{C}-\mathrm{V}$, while the net dielectric charge is simultaneously being controlled. It works as follows: once the charge control loop decides which symbol, BIT0 or BIT1, must be applied during the next sampling period, the values of $V^{+}$and $V^{-}$ are adapted to maintain the target capacitance. That is, at each sampling time, depending on whether $C^{+}$and $C^{-}$are above or below $C_{t h}, V^{+}$and $V^{-}$are increased or decreased in a small voltage step $\Delta V$.

Fig. 3 illustrates qualitatively how this double-loop control works. Two different situations are shown. In the first-initial 


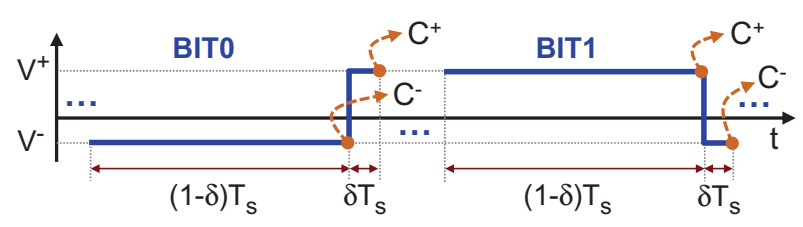

Figure 1. Voltage symbols BIT0 and BIT1 used to actuate the device.

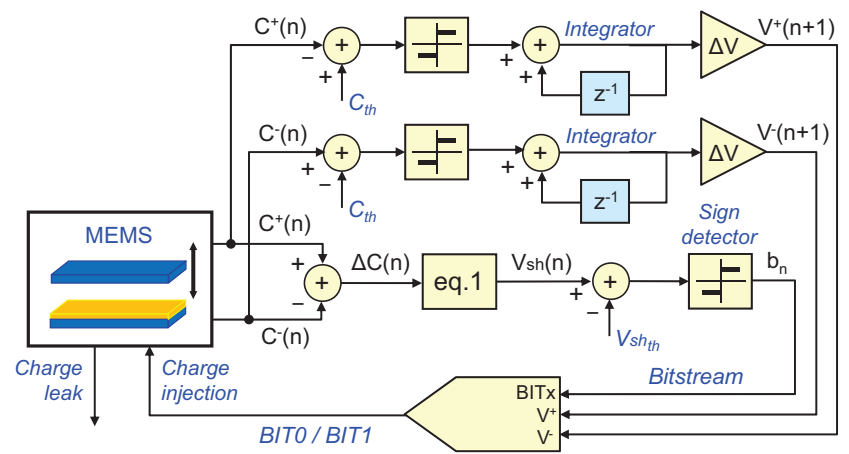

Figure 2. Block diagram of the double-loop control proposed.

one (curve 1), BIT0s and BIT1s are applied to set the bottom of the $\mathrm{C}-\mathrm{V}$ at $V=V_{s h 1}<0$, being $V_{1}^{+}$and $V_{1}^{-}$the voltages needed to obtain the target capacitance $C_{t h}$. In the second case (curve 2), a majority of BIT0s has been applied to shift the curve to $V_{s h 2}>0$. However, since the $\mathrm{C}-\mathrm{V}$ has also suffered some vertical shift, the applied voltages must be set to $V_{2}^{+}$and $V_{2}^{-}$to keep the same device capacitance $C_{t h}$. This means that horizontal displacements produced by net charge injection can be compensated by applying appropriate sequences of BIT0 and BIT1, while vertical displacements of the $\mathrm{C}-\mathrm{V}$ can be compensated through $V^{+}$and $V^{-}$variations.

Summarizing, the double-loop control is applied as follows:

1) Set the values of $\Delta V, T_{S}, \delta, C_{t h}, V_{s h_{t h}}$ and the initial values of $V^{+}$and $V^{-}$.

2) Actuate the device by applying either BIT0 or BIT1.

3) Perform capacitance measurements at symbol times (1$\delta) T_{S}$ and $T_{S}$ and obtain $C^{+}(n)$ and $C^{-}(n)$.

4) Obtain $V_{s h}(n)$ using eq. (1) and decide the next actuation symbol, accordingly to:

$$
B I T(n+1)= \begin{cases}B I T 0 & \text { if } V_{s h}(n)<V_{s h_{t h}} \\ B I T 1 & \text { if } V_{s h}(n)>V_{s h_{t h}}\end{cases}
$$

5) Set the new values of $V^{+}$and $V^{-}$for the next sampling period, by adding or subtracting $\Delta V$, accordingly to:

$$
\begin{aligned}
& V^{+}(n+1)=V^{+}(n)+\Delta V \cdot \operatorname{sgn}\left(C_{t h}-C^{+}(n)\right) \\
& V^{-}(n+1)=V^{-}(n)-\Delta V \cdot \operatorname{sgn}\left(C_{t h}-C^{-}(n)\right)
\end{aligned}
$$

6) Apply the next symbol and go back to step 3 .

\section{EXPERIMENTAL RESULTS}

This section presents experimental results illustrating the performance of the double-loop control proposed. The device used, fabricated with standard PolyMUMPS technology, has a two-parallel plate structure of $515 \mu \mathrm{m} \times 515 \mu \mathrm{m}$ area. The movable plate (top electrode) is a stack of $0.5 \mu \mathrm{m}$ gold and

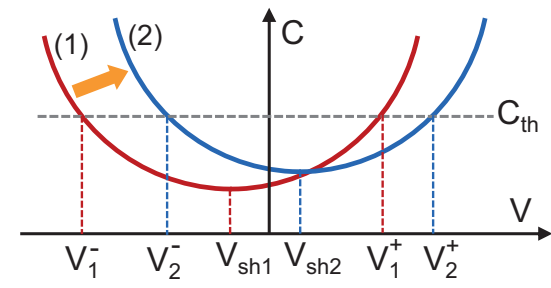

Figure 3. Illustration of hypothetical $\mathrm{C}-\mathrm{V}$ displacements generated by the application of the proposed control method.

$1.5 \mu \mathrm{m}$ polysilicon layers. The plate is held by four L-shaped arms, of $12 \mu \mathrm{m}$ width and $250 \mu \mathrm{m}$ length, over a $2.75 \mu \mathrm{m}$ air gap and a $0.6 \mu \mathrm{m}$ silicon nitride layer deposited on the silicon substrate (bottom electrode). The parasitic capacitance due to pads and tracks is $5.1 \mathrm{pF}$. A precision LCR meter measures the device capacitance and applies the BIT0 and BIT1 voltage waveforms. This equipment is controlled from a computer, which performs data calculations and takes the decisions of the control loops.

In a first experiment, three target capacitances, $C_{t h}=6.34$, 6.36 and $6.35 \mathrm{pF}$, were set in 5-hour steps, while $V_{s h_{t h}}=0 \mathrm{~V}$ (thus zero net dielectric charge) was also set. The time parameters of BIT0 and BIT1 are $\delta=1 / 7$ and $T_{S}=816 \mathrm{~ms}$. The initial voltages are $V^{+}=-V^{-}=7 \mathrm{~V}$ and the voltage step is $\Delta V=10 \mathrm{mV}$. The results, see Fig. 4, show that the double-loop control works successfully, allowing to reach and maintain the three different device capacitance values, while the net dielectric charge is kept constant during the whole experiment. The bitstream generated by the first control loop sets the net dielectric charge while the additional control loops adapt the actuation voltages, $V^{+}(t)$ and $V^{-}(t)$, to keep constant the device capacitance. The time evolution of the averaged bitstream is clearly affected by the evolution of the actuation voltages. This reflects the fact that the dynamics of these three actuation variables are clearly intertwined.

In the experiment reported in Fig. 5 three different strategies were successively applied. The time parameters of BIT0 and BIT1 used are $\delta=1 / 5$ and $T_{s}=1.2 \mathrm{~s}$. For the first 6 hours, the device was actuated with an open loop sequence of alternating BIT0 and BIT1 symbols, with $V^{+}=-V^{-}=6 \mathrm{~V}$. It is clearly observed that neither the device capacitance nor the net dielectric charge are controlled, since the $\mathrm{C}-\mathrm{V}$ suffers from both horizontal and vertical displacements that are not being compensated. In the second stage, from $t=6 h$ to $t=12 h$, the charge control method reported in [3] is used to set zero net dielectric charge $\left(V_{s h_{t h}}=0 \mathrm{~V}\right)$. Fig. 5 shows that this method allows to set the voltage shift to the desired value, thus charge control is achieved, but no capacitance control is obtained. Finally, from $\mathrm{t}=12 \mathrm{~h}$ on the proposed double-loop method with $V_{s h_{t h}}=0 \mathrm{~V}$ and $C_{t h}=6.04 \mathrm{pF}$ is applied and thus both the net charge and the device capacitance are effectively controlled.

Fig. 6 shows the $\mathrm{C}-\mathrm{V}$ curves obtained at the beginning and at the end of the experiment reported in Fig. 5. The initial voltage shift (curve (a)) is $V_{s h}=0.5 \mathrm{~V}$, corresponding to an amount of positive net charge. At $\mathrm{t}=18 \mathrm{~h}$, after applying the 

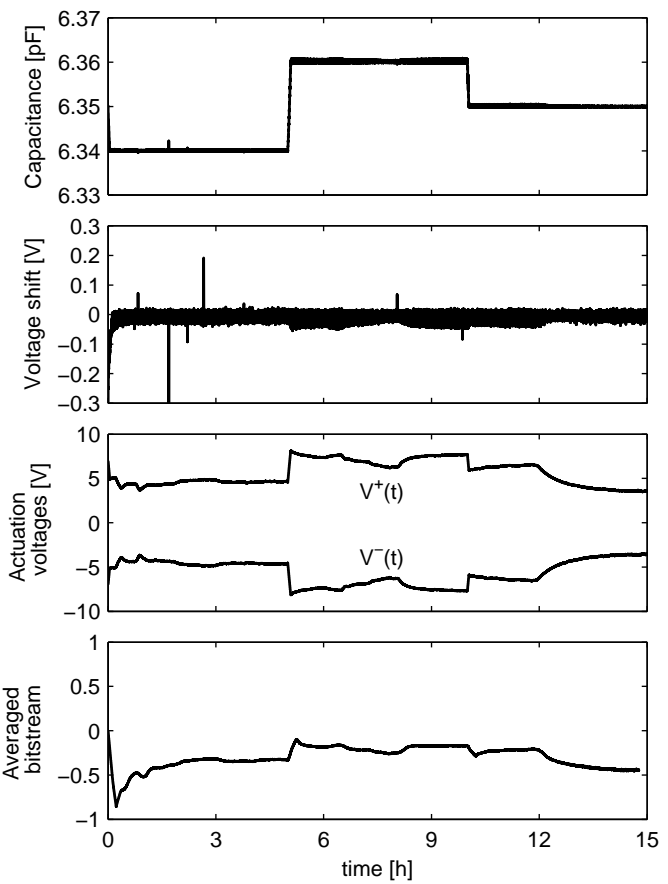

Figure 4. Results of an experiment in which the double-loop control is applied to set zero net charge and three different consecutive target capacitances.

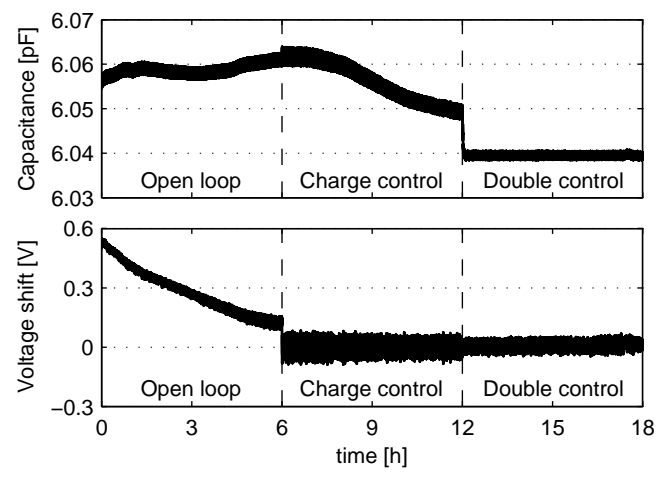

Figure 5. Comparison between three different actuation strategies: open loop, sigma-delta charge control and double-loop control.

double-loop control (curve (b)), $V_{s h}$ and thus the net charge are zero, as expected. Note that although a vertical shift of the $\mathrm{C}-\mathrm{V}$ is observed, it has been compensated by adapting $V^{+}(t)$ and $V^{-}(t)$ to maintain the device capacitance constant.

Finally, the experiment of the first step of Fig. $4\left(C_{t h}=\right.$ $6.34 \mathrm{pF}, V_{s h_{t h}}=0 \mathrm{~V}$ ) was repeated for 36 hours, while the temperature was being monitored. Fig. 7 shows the results obtained, where a clear correlation between the evolution of the temperature and the actuation voltages is observed. As in the case of Fig. 4, the behaviour of the actuation voltages $V^{+}(t)$ and $V^{-}(t)$ is symmetrical, then only $V^{-}(t)$ is plotted in Fig. 7. Since the actuation voltages compensate vertical shifts of the $\mathrm{C}-\mathrm{V}$, we can conclude that in this experiment such vertical shifts are mainly due to temperature variation.

\section{CONCLUSIONS}

A new double closed loop method to set both a net dielectric charge and a device capacitance in contactless electrostatic

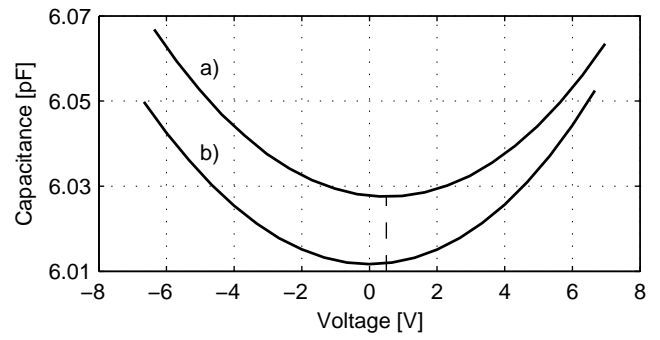

Figure 6. Initial and final C-V curves from the experiment reported in Fig. 5.
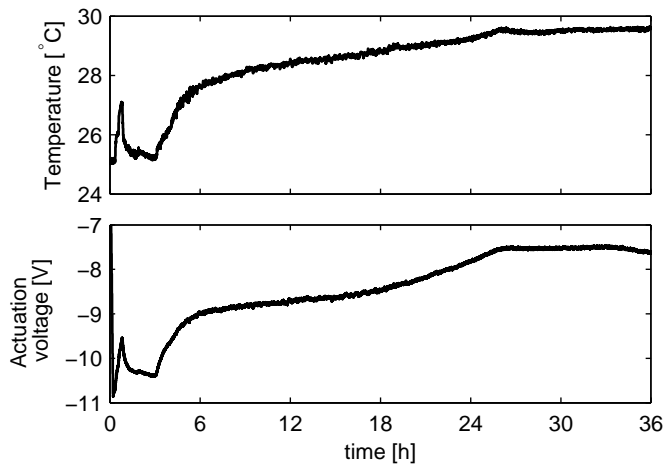

Figure 7. Evolution of the temperature and $V^{-}(t)$ while applying the doubleloop control.

MEMS has been introduced. To keep the capacitance stable at a desired value, the proposed method compensates vertical displacements of the $\mathrm{C}-\mathrm{V}$ by adjusting the actuation voltages. The results obtained demonstrate the goodness of the method to avoid undesired effects caused, among other potential factors, by temperature variation.

\section{REFERENCES}

[1] W. Van Spengen, "Capacitive RF MEMS switch dielectric charging and reliability: a critical review with recommendations," Micromechanics and Microengineering, J. of, vol. 22, 2012.

[2] T. Ikehashi et al., "An RF MEMS variable capacitor with intelligent bipolar actuation," in IEEE Int. Solid-State Circuits Conf. (ISSCC 2008), Feb 3-7, 2008, pp. 581-583.

[3] S. Gorreta, J. Pons, E. Blokhina, O. Feely, and M. Domínguez, "Deltasigma control of dielectric charge for contactless capacitive MEMS," IEEE-JMEMS, vol. 23, no. 4, pp. 829-841, 2014.

[4] S. Gorreta, J. Pons-Nin, E. Blokhina, and M. Dominguez, "A secondorder delta-sigma control of dielectric charge for contactless capacitive mems," IEEE-JMEMS, vol. 24, no. 2, pp. 259-261, 2015.

[5] R. Herfst, P. Steeneken, and J. Schmitz, "Time and voltage dependence of dielectric charging in RF MEMS capacitive switches," in IEEE 45th Int. Reliability Physics Symposium, 2007, pp. 417-421.

[6] X. Rottenberg et al., "Analytical model of the DC actuation of electrostatic MEMS devices with distributed dielectric charging and nonplanar electrodes," IEEE-JMEMS, vol. 16, pp. 1243-1253, 2007.

[7] G. Papaioannou, M. Exarchos, V. Theonas, G. Wang, and J. Papapolymerou, "Temperature study of the dielectric polarization effects of capacitive RF MEMS switches," IEEE Trans. on Microwave Theory Tech., vol. 53, pp. 3467-3473, 2005.

[8] Z. Olszewski, R. Duane, and C. O'Mahony, "Effect of environment humidity on the C-V characteristics of RF MEMS capacitive switch," in 9th Int. Symp. on RF MEMS and Microsystems (MEMSWAVE), 29 June - 4 July 2008.

[9] R. Herfst, P. Steeneken, H. Huizing, and J. Schmitz, "Center-shift method for the characterization of dielectric charging in RF MEMS capacitive switches," IEEE Trans. on Semiconductor Manufacturing, vol. 21, no. 2, pp. 148-153, 2008 